Territorios 31 / Bogotá, 2014, pp. 15-35

ISSN: 0123-8418

ISSNe: 2215-7484

Acción colectiva en las ciudades latinoamericanas

\title{
¿Organizaciones o movimientos sociales? Esbozo de una crítica a una distinción conceptual. El caso de las organizaciones sociales en la Ciudad de México*
}

Organizations or Social Movements? Outline of a Critique of a Conceptual Distinction. The Case of Social Organizations in Mexico City

¿Organizações ou movimentos sociais? Esboço de uma crítica a uma distinção conceitual. O caso das organizações sociais na Cidade do México

\section{Édgar Guerra Blanco **}

"Nadie sabe mejor que tú, sabio Kublai, que no se debe confundir nunca la ciudad con el discurso que la describe. $Y$, sin embargo, entre la una y el otro hay una relación".

Recibido: 2 de agosto de 2014

Aprobado: 2 de septiembre de 2014

Doi: dx.doi.org/10.12804/territ31.2014.01

Para citar este artículo:

Guerra Blanco, E. (2014). ¿ Organizaciones o movimientos sociales? Esbozo de una crítica a una distinción conceptual. El caso de las organizaciones sociales en la Ciudad de México. Territorios, (31), 15-35. Doi: dx.doi. org/10.12804/territ31.2014.01

* Agradezco al evaluador de este artículo por las observaciones y sugerencias que hizo para mejorar este trabajo. Por supuesto, los errores y omisiones son de mi exclusiva responsabilidad.

** Doctor en Sociología por la Universidad de Bielefeld (Alemania). Profesor e investigador del Centro de Investigación y Docencia Económicas A. C. (CIDE), Sede Región Centro, Programa de Politica de Drogas. Correo electrónico: edgarguerra@cide.edu 
Palabras clave

Organizaciones de base, organizaciones populares, movimientos sociales, teoria de sistemas sociales, protesta.

Keywords

Grassroots organizations, people's organizations, social movements, social systems theory, protest.

Palavras-chave

Organizações de base, organizações populares, movimentos sociais, teoria de sistemas sociais, protesta.

\section{territarias 31}

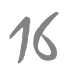

\section{RESUMEN}

Junto con los movimientos sociales, las organizaciones sociales en la ciudad de México han sido actores clave en el impulso, dirección y consolidación de los procesos de liberalización y democratización del sistema político mexicano en general, y, en lo particular, de una buena parte de los cambios políticos y sociales acaecidos en la ciudad capital. Más aún, la complejidad de la Ciudad de México y la variedad de los problemas que contiene es, quizás, un indicador de lo variopinto que se presenta al observador el campo y las redes de organizaciones. Sin embargo, son las organizaciones sociales de base, en el sentido de Castells (1988), que, en algún momento de su existencia, ejercen la protesta como forma de comunicación las que en este artículo nos interesan. La pregunta que queremos responder es si estas organizaciones se deben estudiar como organizaciones o como movimientos sociales y, una vez hecha la elección, si esto implicaría decantarse por las herramientas de las teorías sobre los movimientos sociales o de la sociología de las organizaciones. Para esto, se propone un modelo analítico que, en sus líneas generales, observe el caso como organizaciones en protesta, sobre la base de una teoría de los sistemas sociales. ${ }^{1}$

\section{ABSTRACT}

Along with social movements, social organizations in Mexico City have been key actors in the direction and consolidation of the liberalization and democratization processes of the Mexican political system and its main political and social changes. Moreover, the complexity of Mexico City and the variety of problems in it is, perhaps, an indicator of how diverse it is presented to the observer the field of social organizations. However, this article is concerned in grassroots organizations (Castells, 1988) especially those engaged in the protest as a form of communication. The question to answer is whether these organizations should be studied as organizations or as social movements and, once the choice has been made, if this means deciding between social movement's theories or sociology of organizations. This article proposes an analytical model that looks at the case as organizations in protest, on the basis of a theory of social systems.

\section{RESUMO}

Junto com os movimentos sociais, as organizações sociais na Cidade do México tem sido atores chave no impulso, direção e consolidação dos processos de liberalização e democratização do sistema político mexicano em geral e, no particular, de uma grande parte das mudanças políticas e sociais acontecidos na cidade capital. Ainda mais, a complexidade da Cidade do México e a variedade dos problemas que contém é, talvez, um indicador do heterogêneo que se apresenta ao observador o campo e as redes de organizações. No entanto, são as organizações sociais de base, no sentido de Castells (1988) que, em algum momento de sua existência exercem a protesta como forma de comunicação, as que neste artigo interessam-nos. A pergunta que interessa-nos responder é se estas organizações devem-se estudar como organizações ou como movimentos sociais e quando feita a escolha, se isto implicaria se decantar pelas ferramentas das teorias sobre os movimentos sociais ou da sociologia das organizações para isto propõe-se um modelo analítico que, em linhas gerais, observe o caso como organizações em protesta, sobre a base de uma teoria dos sistemas sociais. 


\section{Introducción}

Una breve mirada a la historia política y social reciente de México mostrará, sin duda, la enorme variedad de formas y contenidos que han adoptado las organizaciones sociales que se han manifestado a lo largo del siglo XX y la primera década del XXI, en el marco de las profundas transformaciones económicas, políticas, sociales y culturales en el país. Desde las organizaciones que apoyaban los movimientos de médicos y maestros durante los años dorados del régimen político mexicano (1940-1960), hasta aquellas que acompañaron las movilizaciones poselectorales del cardenismo en 1988, pasando por el 68 mexicano, las guerrillas urbanas y rurales de los años setenta y el Movimiento Urbano Popular en los ochenta. En todas estas coyunturas, las organizaciones sociales han mostrado características similares en sus formas y estructuras organizativas, en sus demandas y programas, y en los alcances de sus actividades en función de su relación con el régimen político. $\mathrm{Al}$ mismo tiempo, los marcos analíticos empleados en la academia para estudiar esta manifestación de lo social presentaban cierta unidad al enfocar la mirada al estudio de las variables económicas y políticas (desde una perspectiva marxista) de los mecanismos de respuesta a la disidencia implementados por el Estado — como la represión-y a las demandas de los grupos movilizados, entre otros elementos, para entender y explicar a las organizaciones y sus eventos de movilización (Ramírez Sáiz, 1987).

¿ORGANIZACIONES O MOVIMIENTOS SOCIALES? ESBOZO DE UNA CRÍTICA A UNA DISTINCIÓN CONCEPTUAL
Sin embargo, con la irrupción del Movimiento Zapatista y el EZLN en 1994, en el marco de una sociedad mexicana en profundo cambio, ${ }^{2}$ el panorama de las organizaciones sociales no ha cesado de ofrecer sorpresas, que consisten, en lo esencial, de la emergencia de nuevos temas y demandas, formas de organización y movilización e, incluso, de identidades y narrativas que rápidamente se han posicionado en la opinión pública. Así, junto al EZLN, surgieron proyectos desde la sociedad civil, como Alianza Cívica, los cuales parecieron convertirse, en su momento, en los modernos representantes de la sociedad civil mexicana. Además, parecía que la modernización de la sociedad no solo se traducía en las formas en que la sociedad se organizaba y se manifestaba, o en la democratización de las instituciones políticas, en la apertura de las fronteras comerciales y financieras, y en la profesionalización de la ciencia, sino, también, en las formas en que la sociedad se describía a sí misma, puesto que en el discurso público comenzaba el abandono y desuso de nociones que se presentaban ya como 'vetustas' y 'premodernas', por lo que se adoptaba, en cambio, un lenguaje más contemporáneo, en el cual conceptos como 'globalización', 'sociedad civil' y 'activismo ciudadano' - entre muchos otros - reemplazaban los de 'nacionalización', 'clases populares' y 'lucha social'. En este sentido, la emergencia de tales formas de organización trajo consigo, también, nuevos retos académicos, como estudiarlas en su complejidad y en su rol dentro de una sociedad en cambio, lo que detonó la
${ }^{1}$ La introducción de la teoría de los sistemas sociales de Niklas Lubmann en el estudio de la protesta en general y los movimientos sociales en particular no es una nueva tendencia. De becho, el propio Lubmann trató el tema en Lubmann (1996, 2003, 2004, 2007). Así mismo, sus discípulos y colegas han hecho lo propio. Ver, por ejemplo, Ablemeyer (1995), Hellmann (1996), Japp (1986a, 1986b, 1993). El modelo más contemporáneo y, a mi parecer, el más comprehensivo para analizar los movimientos sociales desde la perspectiva de los sistemas sociales lo desarrolla Estrada Saavedra (2012a, 2012b y 2013). Lo que aqui se presenta aborda a las organizaciones sociales como sistemas sociales.

${ }^{2}$ Cambios en casi todas las dimensiones de la sociedad y que han sido profusamente estudiados como la transición politica (Cansino Ortiz, 2000; Crespo, 2003), el cambio de modelo económico y sus efectos (Calva, 2001), asi como la transformación de la sociedad civil (Olvera Rivera, 1998).

\section{territarias 31}

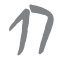


adopción de nuevos enfoques, el giro hacia la investigación de múltiples dimensiones analíticas, así como el empleo de instrumentos conceptuales alternativos.

No obstante, en el México contemporáneo, ambas formas de organización social - la 'popular' y la acuñada desde la 'sociedad civil' - se mantienen vigentes. Más aún, ambas han sido clasificadas, tanto en el discurso político y mediático como en el científico, en los extremos opuestos de un continumm que discurre, en términos generales, desde una concepción de la sociedad como tradicional, en la que se encuentran las organizaciones populares, hacia la idea de una sociedad moderna, de la que surgen las organizaciones de la sociedad civil. Sin embargo, sociológicamente, ambas formas de organización presentan, al menos, tres características comunes: 1) estructuras y dinámicas organizacionales internas; 2) relaciones de cooperación o conflicto con su medio ambiente; y 3 ) el uso recurrente o sistemático de acciones de protesta para influir en el sistema político. De ahí que ambos tipos se puedan unificar como un mismo objeto de estudio: como organizaciones sociales que ejercen la protesta como principal medio de comunicación con su entorno. No obstante, y he aquí nuestro interés principal, cabe la pregunta: ¿se trata de organizaciones, de movimientos sociales o de organizaciones de movimientos sociales? Responder a esta interrogante resulta fundamental para el análisis de este tipo de organización, pues este se encuentra en la frontera entre la organización y la protestersitarios 31 18 con las herramientas de la sociología de las organizaciones, ya con los instrumentos de las teorías sobre los movimientos sociales. Sin embargo, estudiar estas organizaciones exclusivamente como organizaciones empobrece el debate, porque deja de lado los aspectos contenciosos; mientras que estudiarlas como movimientos carga la discusión hacia el aspecto normativo y coloca esperanzas utópicas en actores que, antes que nada, son organizaciones con objetivos y funciones delimitadas. De ahí, nuevamente, la necesidad de encontrar el justo medio con una perspectiva sistémica.

El dilema consistió, por lo tanto, en ubicar esta propuesta analítica o dentro del área de los estudios organizacionales, la cual se ocupa de la forma en que los individuos construyen y actúan en procesos, estructuras y prácticas organizacionales, al tiempo que observa cómo las organizaciones reestructuran las relaciones sociales entre los individuos (ver Clegg \& Bailey, 2008), o dentro del área de los movimientos sociales, la cual centra su mirada en los aspectos contingentes, informales y contenciosos de la acción colectiva (Porta \& Diani, 2005). No obstante, en el transcurso de la investigación, pronto fue claro que, en lo particular, dos perspectivas teóricas permitirían investigar a profundidad las relaciones entre organización y protesta: las teorías de los movimientos sociales y la teoría de los sistemas sociales en la versión de Niklas Luhmann. En efecto, las teorías de los movimientos sociales preguntan sobre la relación entre escasez, agravios y protesta, y las formas de organización, 
movilización y manifestación pública del descontento. Por su parte, la segunda perspectiva teórica, la de los sistemas sociales, entiende a las organizaciones como sistemas sociales que se distinguen a través de expectativas formalizadas de membrecía (Luhmann, 1964) y que operan dentro de un entorno social diferenciado en diversos sistemas de funciones (Luhmann, 1977). En las siguientes secciones, mostraré, precisamente, cómo estudiar las organizaciones sociales que ejercen la protesta desde una perspectiva sistémica. Y es que, si bien se han sentado las bases teóricas para estudiar el tipo de organización que aquí interesa, falta sistematizar la información en un esquema unitario.

De ahí la pertinencia de este esbozo analítico, el cual tiene como base la investigación empírica. En efecto, a través de un proyecto de investigación que emprendí, en el verano de 2009, sobre organizaciones y movimientos sociales en la Ciudad de México, busqué ofrecer una descripción de uno de los campos organizacionales que se ha configurado alrededor de los partidos políticos en la ciudad, principalmente el Partido de la Revolución Democrática y el gobierno de la Ciudad de México, ${ }^{3}$ y en el cual se encontraban tanto organizaciones populares como organizaciones de la sociedad civil que hacían uso sistemático de la protesta. El objetivo fue observar, entre otras dimensiones, cómo los miembros de tales organizaciones construyen y actúan dentro de procesos, estructuras y prácticas organizacionales y de protesta, y cómo las organizaciones han reestructurado las relaciones sociales entre sus miembros, incluso más allá del espacio propiamente organizacional, en un ambiente de protesta y conflicto frente al sistema político (Guerra Blanco, 2012, 2013b, 2014).

Si bien estas organizaciones populares y de la sociedad civil han merecido diversos estudios sobre su capacidad de apoyo electoral al sistema de partidos políticos, sobre su capacidad de protesta frente al gobierno de la ciudad y sobre la red corporativista y clientelar que han construido (Olvera Rivera, 2001; Álvarez Enríquez \& Bolos, 2003; Álvarez Enríquez, San Juan Victoria \& Sánchez Mejorada, 2006; Olvera Rivera, 2004), ${ }^{4}$ aún no se repara en la necesidad de diferenciar conceptualmente entre organización y movimiento, y elaborar una propuesta que permita el análisis interno de tales organizaciones en protesta. La propuesta analítica fue, por lo tanto, resultado de mi interés por los estudios de protesta social en espacios urbanos y, de forma específica, por los roles que juegan las organizaciones en la movilización de los recursos humanos, materiales y simbólicos. Sin embargo, el proyecto dio un paso más allá de las coyunturas de la protesta y conflicto para observar qué ocurría dentro de las organizaciones, cómo se acoplaban a las diversas dimensiones de la sociedad moderna y en qué sentido la vida dentro de las organizaciones impactaba tanto en el hombre organización (Whyte, 2002) como en la sociedad organizada.

El modelo se pensó para analizar un mismo campo organizacional. Dentro de este campo, algunas de las organizaciones
${ }^{3}$ Me refiero al entramado de organizaciones sociales, popularesy de la sociedad civil que se ha desarrollado al oriente de la Ciudad de México, en la delegación politica de Iztapalapa (ver, por ejemplo: Paladino, 2010).

${ }^{4}$ Históricamente, entre las tareas más memorables de las organizaciones sociales defeñas se hallan, desde la organización colectiva de los habitantes de la ciudad con el objetivo de asegurar espacios habitacionales, servicios urbanos o ejercer sus derechos ciudadanos, hasta la movilización de la militancia frente a contingencias y desastres naturales, así como para emprender campañas de promoción política y defensa del voto o de rescate del entorno urbano.

territarias 31

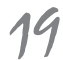


pertenecen, por sus orígenes, al Movimiento Urbano Popular, otras más son de relativa nueva creación y, en especial, son resultado de las políticas públicas, corporativas y clientelares del gobierno de la ciudad, el cual incentiva la organización social como un mecanismo de apoyo político y electoral a cambio de prebendas y programas sociales de desarrollo. Cada organización es autónoma en sus operaciones; cada una despliega sus propios programas, objetivos, formas de acercarse a las bases, etc. No obstante, en conjunto, forman un campo que comparte definiciones comunes $\mathrm{o}$, al menos, compatibles - o no excluyentes- de la realidad, formas conocidas y usuales de la lucha y de la organización social, así como culturas organizacionales similares y formas semejantes de relacionarse con la política.

\section{1. ¿Las organizaciones sociales, populares y de la sociedad civil son organizaciones?}

Las organizaciones son, de forma indiscutible, un rasgo característico de la sociedad moderna, la cual, además, define y determina su funcionamiento. De ahí que, junto a los usuales conceptos para definir a la sociedad contemporánea (sociedad de la información, posindustrial, posmoderna, del riesgo, del conocimiento), la moderna sea una sociedad que haya sido definida, también, como una sociedad de organizaciones (ver Abraham \& Büschges, 2004; Gabriel, 1979; Mayntz, 1971; Perrow, 1991; Presthus, 1962; Simon, 1991). Si bien la sociedad moderna no es una organización y no todas las relaciones sociales que la cruzan son relaciones propias de las organizaciones (ver Luhmann, 1975; 1981, p. 391), las formas que la sociedad moderna presenta para organizarse o para construir organizaciones se despliegan a lo largo de todos los ámbitos de la sociedad; es decir, casi ninguna dimensión social escapa a las organizaciones. Así, en la política, se forman partidos; en la ciencia, universidades y centros de investigación; en la economía, bancos, empresas mercantiles; en el ámbito de las relaciones amorosas, aparecen agencias matrimoniales; $y$, en la protesta social, surgen organizaciones, así como también existen organizaciones sociales que deben ejercer la protesta para garantizarse recursos y lograr el cumplimiento de sus objetivos. De hecho, es tan amplia y profunda nuestra relación con las organizaciones que los hombres pasan la mayor parte de su tiempo en ellas (ver March \& Simon, 1958, p. 2).

Las organizaciones sociales, populares y de la sociedad civil son, también, organizaciones, como lo son las universidades y centros de investigación, las iglesias, los bancos o los museos. Como cualquier organización, las organizaciones sociales, populares y de la sociedad civil: 1) mantienen una membresía o una planta de militantes; 2 ) operan en aras de lograr objetivos programáticos y alcanzar metas estratégicas; 3 ) cumplen ciertas funciones tanto a nivel interno como en un entorno que consiste de otras organizaciones; 4) desarrollan sus propios programas y objetivos, estrategias y tácticas, identidades, descripciones de 
sí mismas y de su entorno; 5) así como sus propias estructuras de posiciones, las cuales, a veces, presentan la forma de jerarquía y, otras tantas, formas horizontales; 6) desarrollan sus mecanismos internos de participación y solución de conflictos que o bien transmiten decisiones desde un ápice hacia las bases o fomentan la formación de consensos y decisiones democráticas desde la base hasta la dirección. 7) Además de generar un ambiente interno con relaciones sociales específicas y su propia cultura organizacional, 8) las organizaciones operan en un entorno social, que es propio del ámbito de sus actividades, pero que se amplía en función de la complejidad estructural interna y de los propios requerimientos de estas. Así, por ejemplo, una organización de la 'sociedad civil' no solo efectúa operaciones políticas, sino que también establece vínculos operativos con el sistema económico, legal, educativo, de salud, etc. 9) En este sentido, las organizaciones devienen en actores (Hutter \& Teubner, 1994) que contribuyen a las operaciones de los sistemas funcionales de la sociedad moderna. 10) Finalmente, las organizaciones que en este manuscrito se proponen como caso son aquellas que hacen un uso sistemático e intenso de la comunicación de protesta para enlazarse o entablar relaciones con el sistema político. De ahí, precisamente, su carácter contencioso, lo que les da una forma singular y, por momentos, ambigua. En fin, estas son, en diez puntos, las características que convierten a las organizaciones sociales, tanto populares como de la sociedad civil, en organizaciones o sistemas sociales con la forma organización. De ahí que proponga estudiarlas como organizaciones en protesta y no tanto como movimientos de protesta (Luhmann, 1996) o sistemas de protesta (Hellmann, 1996; Estrada, 2013), ya que el uso que estas organizaciones hacen de la protesta es con miras al cumplimiento de fines organizativos. ${ }^{5}$ Sin embargo, el hecho de que, efectivamente, hagan uso recurrente de la protesta nos conduce a preguntarnos cómo se ha tratado el tema de la organización y la protesta en la literatura actual.

\section{Protesta y organización, la discusión actual}

A primera vista, pareciera que el campo de investigación sobre los movimientos sociales, por un lado, y aquel sobre las organizaciones, por el otro, tuvo un desarrollo paralelo con breves y escasos momentos de convergencia. Por ejemplo, los estudios organizacionales se centraron en las organizaciones con una estructura formal y jerárquica, con objetivos programáticos sumamente delimitados y definidos, mecanismos formales y burocráticos para la toma de decisiones y la ejecución de sus operaciones en un contexto de relativa estabilidad y adaptación a sus entornos (Porta \& Diani, 2005 ). Por otro lado, la investigación empírica sobre los movimientos sociales enfatizó su estructura informal y horizontal, la contingencia de sus objetivos y estrategias que se redefinen al calor de las luchas, las estructuras y mecanismos horizontales para la toma de decisiones y su permanencia
${ }^{5}$ En este sentido, es ejemplar el caso del Frente Popular Francisco Villa, una organización popular militante que nació con un proyecto politico y social: por un lado, el objetivo estratégico de transformar al pais por la vía del socialismo y, por otro, metas tácticas que consistían, en lo esencial, en encontrar soluciones a una serie de problemas urbanos, como el de vivienda, que le aseguraran una amplia base social. Con este fin, la organización hizo uso recurrente de la protesta para, así, exponer su problemática social, adquirir recursosgubernamentales a través de politicas públicas o asignación directa y, con el paso del tiempo, lograr posiciones políticas en alianza con losgobiernos locales de izquierda. Esdecir, al finaldel camino, la protesta y movilización devino en una estrategia para el intercambio de recursos organizativos y políticos.

\section{territarias 31}

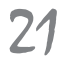


- Sin duda alguna, tanto la teoría de los 'nuevos movimientos sociales'o 'paradigma orientado a la identidad' (Cohen, 1985) como las teorias culturalistas y constructivistas de los movimientos sociales (Laraña, 2007) aportan un enorme acervo de conocimiento al estudios de las formas de organización más desestructuradas que emergen dentro de las dimensiones culturales, discursivas y simbólicas de la movilización y la protesta.

7 No es posible aquí, por cuestiones de espacio, elaborar una revisión sistemática y pormenorizada de las diferentes propuestas teóricas que abordan, de una $u$ otra forma, la relación entre organización y protesta. Sobre el tema, ver Guerra Blanco (2013a). En especial, el primer capitulo.

\section{territarias 31}

en un entorno de incertidumbre cargado de disrupción y contención (Davis et al., 2005 ). No sin razón, algunos analistas han criticado la división radical entre ambos campos, haciendo notar que, mientras los estudios organizacionales centran sus investigaciones en la acción instrumental y organizada, la investigación en movimientos sociales se ha centrado en fenómenos menos estructurados, espontáneos y desorganizados (Morris, 2000), así como lo ha hecho el paradigma de los 'nuevos movimientos sociales' ${ }^{6}$

Tales distinciones analíticas parecen tener su base en las evidentes características del objeto de investigación, es decir, en las organizaciones formales y en los movimientos sociales. Pero poner las principales distinciones y fronteras entre los estudios organizacionales y la investigación sobre los movimientos sociales en una oposición tan radical es, sin embargo, un mal entendido. Desde los primeros desarrollos teóricos, la teoría de la movilización de recursos centró sus esfuerzos analíticos en la observación y desarrollo de las organizaciones de los movimientos sociales (McCarthy \& Zald, 1990). Hubo, de hecho, procesos fructíferos de irritaciones mutuas que impactaron el desarrollo teórico. Incluso, tal como la posterior literatura y crítica a la perspectiva de movilización de recursos muestra, algunos de los principales conceptos y estrategias analíticas de la movilización de recursos provienen directamente de los estudios de administración y organizaciones (Jenkins, 1983). Por otro lado, la literatura reciente, si bien no soslaya la diferencia entre ambos objetos de estudio, entre organizaciones y movimientos sociales, sí observa temas comunes, convergencias teóricas y campos homogéneos para la investigación empírica y la correspondencia conceptual, lo cual se ha profundizado con el reciente giro hacia la perspectiva de los sistemas abiertos dentro de los estudios organizacionales (Davis et al., 2005). Es decir, en las teorías clásicas de los movimientos sociales, el tema de la organización ha sido omnipresente.

En efecto, cualquier revisión somera de la literatura científica muestra la larga tradición de investigación empírica y desarrollo teórico en el tema de los movimientos sociales y su organización, si bien el énfasis del análisis se ha cargado más al estudio del movimiento, como movimiento, y no tanto a las organizaciones - con la excepción de la teoría de movilización de recursos-. No obstante, no hay evidencia de un tratamiento de las organizaciones sociales que recurren a la protesta como medio de $\mathrm{CO}^{-}$ municación con el sistema político, como ocurre en nuestro caso. Con todo, en esta línea de investigación, han proliferado diversas y sumamente diferenciadas teorías y perspectivas analíticas, lo cual ha resultado en un sumamente complejo -y difícil de englobar- conglomerado de teorías, métodos, hipótesis y correlaciones. ${ }^{7}$ En efecto, la mayoría de los modelos existentes en el mercado, que buscan observar, describir y explicar la protesta y su organización, en diferente grado de amplitud y profundidad, han elaborado desde modelos descriptivos hasta teorías generales de la sociedad, pasando por teorías de alcance intermedio. 
Algunas veces, el énfasis de la explicación se ha puesto en las condiciones estructurales de la sociedad (la economía, el sistema político), como es el caso de las teorías de la sociedad de masas y del comportamiento colectivo (Kornhauser, 2008; Turner \& Killian, 1972; Smelser, 1966). Otras tantas, se ha puesto atención en el actor racional y sus intereses, competencias y recursos, como es el caso del modelo de movilización de recursos y el proceso político (McCarthy \& Zald, 1990). Finalmente, se ha privilegiado el análisis de los valores y las normas, así como de las dimensiones cultural, discursiva y simbólica de la acción, para observar los procesos de estructuración organizativa de la protesta, como ocurre en las teorías de los nuevos movimientos sociales o paradigma de la identidad (Cohen, 1985) y las teorías constructivistas (Laraña, 2007). En otros casos, no solamente se ha aceptado y emprendido el riesgo de elaborar síntesis teóricas (Cohen, 1985) a partir de los modelos existentes y con el cúmulo de datos disponibles, sino que, también, se ha buscado construir paradigmas alternativos que sustituyan a los clásicos en el campo (Hellman, 1996). Sin embargo, el resultado final ha sido un laberinto: conceptos, estrategias de investigación, metodologías y teorías; todos con diversas y hasta contradictorias posiciones sobre la ciencia y la realidad, sobre la naturaleza humana, el sentido de la historia y sobre la estructura, dinámicas y fines de la protesta y sus dinámicas organizacionales. Y este laberinto deviene cada vez más difícil de asimilar si, además, observamos que las teorías de los movimientos sociales cambian a la par que la protesta y la sociedad cambian.

Pero, si esto sucede en el plano teórico, habrá que preguntarse, también, ¿̇cómo se han estudiado las organizaciones en protesta en México? Para algunos observadores, la actividad de las organizaciones sociales, especialmente la transformación y evolución que han experimentado a lo largo de la historia reciente, y que va desde la protesta y la movilización social hasta su reciente inclusión en las dinámicas de la política institucional, es un proceso que tiene que explicarse como parte de la constitución de la sociedad civil y como parte de la transición hacia la democracia en México. Ambos cursos históricos han sido investigados desde diferentes perspectivas (Olvera Rivera, 2001; Álvarez Enríquez \& Bolos, 2003; Álvarez Enríquez, San Juan Victoria \& Sánchez Mejorada, 2006; Olvera Rivera, 2004). En términos generales, el argumento señala la mutua dinámica entre las limitaciones estructurales, por un lado, y las oportunidades coyunturales, por el otro, sobre la base de las acciones individuales y colectivas de los actores. La hipótesis principal desde esta perspectiva de investigación es que la acción de las organizaciones y movimientos de la sociedad civil detonó e impulsó el proceso de liberalización y democratización del régimen político, lo cual redundó en nuevas formas de participación e inclusión políticas, que, a su vez, fortalecieron y ampliaron la envergadura de la sociedad civil y sus actores. 
${ }^{8}$ Al respecto, ver la crítica de Estrada \& Guerra (2012) en la que se cuestiona el modelo del actor y se plantean preguntas acerca de su utilidad, en el marco de los avances teóricos recientes, para la investigación empirica. Estrada Saavedra, por su parte, amplía y profundiza la critica con miras a elaborar un modelo analítico de la protesta (2013).

\section{tersitarias 31}

\section{Crítica}

No obstante, a pesar de la pluralidad y diversidad de los modelos de acción colectiva y la organización de protesta, todas las perspectivas descansan en un concepto ontológico de actor. De hecho, esta enorme diversidad de miradas teóricas solo es posible sobre la base de un elemento común que brinda unidad: la acción. Sobre la base de la dupla sujeto/acción, las teorías de los movimientos sociales elaboraron un acercamiento al fenómeno de la protesta y la organización, mediante la observación de la acción colectiva y su racionalidad. Si bien cada perspectiva y giro teórico gozó de cierta autonomía, que permitió un fluido intercambio y crítica, lo cual resultó en el desarrollo de perspectivas más abarcadoras que reconciliaban ciertos antagonismos, los resultados continúan generando controversias y preguntas que aterrizan, precisamente, en el cuestionamiento al estatus ontológico del actor y su acción, el cual está en la base del concepto de acción colectiva contenciosa y movimiento social. ${ }^{8}$

Las teorías sobre protesta y su base epistemológica anclada en el concepto de actor, así como una específica idea de ciencia social (mecanicista y objetiva) sobre la que se construyen las explicaciones del fenómeno, han sido duramente criticadas a la par del desarrollo de la teoría social en general y de las teorías de los movimientos sociales en particular. Sin embargo, recientemente, la crítica se ha focalizado en el concepto de actor. Relevante en este sentido ha sido el giro teórico que los prin- cipales representantes de la perspectiva del proceso político han elaborado sobre el tema. En efecto, en el libro Dynamics of contention (McAdam, Tarrow \& Tilly, 2001), los autores critican todo el paradigma de análisis de la acción colectiva contenciosa para proponer un giro teórico y epistemológico radical. En términos generales, el blanco principal de su ataque es la lógica de explicación de lo social, la cual se centra en la búsqueda de relaciones causales, por un lado, y en el concepto de actor, el cual es un concepto que limita la explicación de la protesta social. Los autores sugieren hacer uso del paradigma de los mecanismos, como el marco principal de explicación, así como del principio de la 'persuasión relacional' como una estrategia para redefinir a los actores y no considerarlos más, como seres o entidades, con estatus ontológico, sino como una 'construcción contingente'. Más allá de la crítica que se puede hacer a su propuesta, es valioso anotar los resultados de este proceso reflexivo dentro de la teoría de los movimientos sociales. Más aún, la crítica que elaboran va directo al corazón de los principales supuestos de las teorías previas sobre los movimientos sociales. De ahí su pertinencia.

$\mathrm{Y}$ es que, en efecto, la influencia del paradigma del actor aparece en todas las teorías de los movimientos sociales. Estas 1) parten de un modelo de contención y organización basado en la acción; 2) muestran una fuerte tendencia normativa en la idea de sociedad civil, pues las organizaciones y los movimientos se aparecen como actores en protesta con miras a liberar y 
democratizar el sistema político y, en este sentido, ensanchar las condiciones de la inclusión política, lo cual, de ninguna manera, es una característica intrínseca a las organizaciones sociales; 3 ) el sistema político aparece como un elemento determinante y sumamente importante en la explicación de la transformación de las organizaciones desde el campo de lo social, hasta devenir en actores y organizaciones políticas, por lo cual se deja de lado el análisis de la dinámica interna y el andamiaje estructural de estas organizaciones (más aún, estas explicaciones de las organizaciones no ofrecen una descripción detallada y densa de los procesos internos que ocurren en las organizaciones y movimientos); 4) finalmente, en estas explicaciones hay siempre una confusión conceptual entre organización como organización y como actores. De ahí que, en efecto, los conceptos como acción colectiva y movimiento social producen imágenes ingenuas del objeto, de su supuesta unidad, coherencia y transparencia como actores colectivos que gozan de una historia, identidad, voluntad, fines y estrategias (Estrada Saavedra \& Guerra Blanco, 2012).

Sin embargo, muchas de estas suposiciones y paradojas que genera el modelo del actor tal vez encuentren una solución si entendemos los movimientos sociales y, especialmente, la organización, no de acuerdo con los modelos de acción social, sino como sistemas sociales. En este caso, es necesario eliminar los conceptos de actor y acción, y reemplazarlos por los de actor ficticio (Hutter \& Teubner, 1994) y atribución (Luhmann, 1987), un punto de partida que conduce hacia una crítica radical de las principales supuestos, conceptos, coherencia interna y poder explicativo de estos modelos. Nuestro punto de partida supone un cambio conceptual de la definición analítica del fenómeno social que se investiga: por un lado, la idea de que las organizaciones en protesta tienen un estatus ontológico como actores colectivos; $y$, por otro lado, un potencial moral para la transformación de la sociedad. En resumen, el desafío consiste no en pensar en la acción colectiva en términos de actor y acción, sino en términos de comunicación. Organizaciones y movimientos como sistemas de comunicación, por un lado, y el actor como una 'construcción comunicativa' o 'actor ficticio', por otro lado.

El giro teórico implica ir más allá de la falsa oposición entre actor y sistema y, en particular, pensar la sociedad en términos de sistemas sociales.

\section{Organización como sistema social}

En esta propuesta, asumimos el giro sistémico para el estudio de las organizaciones sociales, que hacen uso de la movilización contenciosa para formular sus demandas y presentarlas ante las autoridades de gobierno y, de esta forma, encontrar respuesta a sus reclamos. ${ }^{9}$ En este sentido, entendemos estas organizaciones como organizaciones en protesta. Lejos de asumir la ilusoria idea de las organizaciones sociales como un 'hecho social', partimos de organizaciones como una construcción comunicativa, $\mathrm{CO}^{-}$
${ }^{9}$ La etiqueta de giro sistémico en el estudio de los movimientos sociales se refiere, por un lado, a la reformulación del estudio de los movimientos sociales que Niklas Lubmann emprendió en diferentes etapas de su carrera. La lectura, desde un punto de vista sistémico, de las teorias sobre los movimientos sociales fue proseguida, entre otros, por Ablemeyer (1995), Japp (1986a, 1986b, 1993), Lubmann (1996) y Hellmann (1996). Estos autores aportan una literatura esencial para una lectura sistémica sobre los movimientos sociales. Por otro lado, la idea del giro sistémico refiere a la adopción y reformulación que, desde el punto de vista de la teoría de sistemas, se elabora de los conceptos, dimensiones analiticas y categorias de las diferentes teorías convencionales sobre los movimientos sociales. Uno de los esfuerzos más recientes $y$ completos en este sentido es el de Estrada Saavedra (2013).

\section{territarias 31}

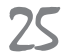


${ }^{10}$ Las organizaciones, como las organizaciones populares de la Ciudad de México, producen de forma intermitente decisiones de todo tipo: deciden los objetivos organizativos (transformación del régimen politico o resolución de demandas sociales), las estrategias y tácticas para alcanzar susmetas (movilización de masas o apoyo a partidos politicos) o, incluso, los propios mecanismos de toma de decisión (elecciones democráticas internas o elecciones unipersonales de los lideres).

\section{territarias 31} 26 mo un orden emergente que surge a partir de la clausura operativa de comunicación y pensamiento, y que trasciende las mentes $\mathrm{y}$ acciones individuales.

Desde la perspectiva analítica que se ha usado en el estudio de organizaciones sociales, los conceptos de protesta y organización refieren exclusivamente a la producción de comunicaciones orientadas a la construcción de decisiones y a la comunicación del conflicto. En este sentido, la organización como sistema debe entenderse, fundamentalmente, como una máquina que produce una particular forma de comunicación: decisiones (Nassehi, 2005), es decir, como una cadena recursiva de decisiones que da curso a la complejidad social, detona procesos de construcción de sentido y estructuras que conforman una cultura organizacional y una estructura organizativa - la organización de la organización-, la cual adquiere diversas formas - como una jerarquía, por ejemplo- y permite a la organización vincularse, de distintas maneras, con su entorno.

\subsection{Las organizaciones como cadenas de decisiones}

En la versión luhmanniana de la teoría de los sistemas, las organizaciones son un tipo de sistema social, junto a las interacciones, los movimientos de protesta y la sociedad (Luhmann, 1998). Para mantenerse como sistemas sociales en diferencia con los otros tres tipos, las organizaciones utilizan sus propios criterios de selección para conectar sus comunicaciones y para establecer sus límites. Como sistemas sociales, las organizaciones consisten en comunicaciones; comunicaciones que se entrelazan constituyendo un entramado operativamente clausurado y autorreferencial. En este sentido, las organizaciones no se diferencian en lo absoluto de los movimientos de protesta o de las interacciones. El elemento que las distingue es, sin embargo, el tipo de comunicaciones que las constituyen. A diferencia de las interacciones sociales que se construyen a partir del uso recursivo de numerosos y variados temas de conversación ( es monótona y busca alargarse), las organizaciones se ocupan de la producción de decisiones. Más aún, las decisiones no solo refieren a las actividades cotidianas propias de cada organización. Dado su carácter autorreferencial, las organizaciones recurren a la producción de decisiones que refieren a decisiones previamente tomadas (Luhmann, 2000, p. 63). Así, la comunicación de decisiones establece, en cada operación, un amplio horizonte de posibilidades que, por sí mismo, compele a la toma de más decisiones. Este proceso desemboca en la emergencia de una cadena. ${ }^{10}$ Las decisiones, al mismo tiempo, son el elemento central que permite construir mecanismos para absorber contingencia, manejar complejidad y riesgos, así como para definir el entorno en el cual se desenvuelven y el tipo de membresía que requieren (Luhmann, 2000, p. 279).

Así, como sistemas sociales autorreferenciales, las organizaciones sociales definen sus operaciones al aplicar, reflexivamente, decisiones sobre decisiones que, 
además, constituyen el punto de partida para la subsecuente toma de decisión. Tal forma de conectividad de sus comunicaciones torna a las organizaciones en sistemas sociales autónomos, al nivel de sus operaciones, y les permite construir complejidad interna. Más aún, si bien tales decisiones se han tomado dentro de un horizonte amplio de posibilidades, como sistemas sociales, las organizaciones hacen uso de un abanico relativamente reducido de operaciones, pues no todas las decisiones son posibles a un mismo tiempo. Solo cierto tipo de decisiones son, finalmente, las que se realizan. Lo anterior sucede porque las organizaciones están constituidas por un marco de expectativas que reduce, direcciona y estimula la decisión que se ajusta más a las expectativas de las organizaciones (Luhmann, 2000, p. 222). El marco de expectativas que configura a toda organización social es la estructura que contiene el abanico de decisiones posibles y, fundamentalmente, probables. En este sentido, y contrario a las teorías convencionales, en la visión luhmanniana es necesario dirigir la mirada tanto a las metas y objetivos de la organización como al marco de expectativas a partir de las cuales aquellos se estructuran (Luhmann, 2005). ${ }^{11}$

En efecto, dado que las organizaciones deben operar sobre la base de ciertas certezas, que son necesarias para la toma de decisiones, las organizaciones aseguran la producción de esas mismas certezas. El primer paso en esta dirección es la configuración de los objetivos y metas de la organización, así como de los medios para cumplirlos, co- mo la construcción de un régimen político alternativo a través de un movimiento de masas. Si bien las metas de cualquier organización no prescriben su función social, sí tienen una función regulativa: ayudan a determinar las reglas internas de operación, los mecanismos de control y el diseño organizativo (Luhmann, 2000, p. 265), por lo que, por ejemplo, si el objetivo último es de naturaleza política y de transformación radical, es probable la configuración de organizaciones jerárquicas y cerradas. Objetivos tales como dar solución a una serie de problemas sociales o la búsqueda de una transformación política radical han sido, sin duda, propósitos fundamentales en la configuración de las identidades y autodescripciones como organizaciones. Sin embargo, los objetivos también cambian a través del tiempo o adquieren una relevancia distinta dentro de la jerarquía de prioridades de la organización. Por lo tanto, en esta propuesta y con el objetivo de lograr un punto de observación desde el cual describir las organizaciones sociales que nos interesan, se toma en cuenta la red de diferencias que están implicadas en la producción de comunicaciones del sistema en diversos espacios de la organización. Distinciones tales como hombre/mujer, miembro/persona, fin/medio, estrategia/ táctica, líderes/miembros, democracia/ autoritarismo, jerarquía/horizontalidad, joven/viejo, social/político, valores/intereses son diferencias que tienen una función de definición en la organización de su propia información. ${ }^{12}$ De tal modo, diversas estructuras se desarrollan para generar
${ }^{11}$ En nuestro caso, este marco de expectativas se configura en dos dimensiones: una dimensión politica que se establece con el objetivo de lograr la transformación del pais hacia el socialismo; y una dimensión social que apunta a la resolución de problemas sociales urbanos.

${ }^{12}$ En efecto, si uno revisa programas de organizaciones populares y sociales de la Ciudad de México, es recurrente la toma de posición sobre diversos temas.

\section{territarias 31}

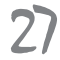


información y seleccionar la más relevante y útil de las decisiones. En estas operaciones, algunas expectativas se solidifican y adquieren la forma de estructuras de expectativas, las cuales contienen sus propios significados.

\subsection{Organizaciones, estructuras y semánticas}

Como sistemas sociales, las organizaciones son tan complejas que necesitan restringir las posibilidades de sus propias operaciones. En este sentido, algunos artefactos están diseñados para evitar los errores en la ejecución de decisiones o para fomentar la conectividad entre las decisiones; y esos artefactos son la red de expectativas que se encuentran integradas en las organizaciones en la forma de estructuras de expectativas. Estas redes de expectativas o estructuras de expectativas tienen la función de regular las comunicaciones organizacionales; por ejemplo, el programa de una organización es una estructura de expectativas que vincula las diversas decisiones que deben tomarse a lo largo del tiempo. Así, el programa político de una organización como el Frente Popular Francisco Villa enlaza objetivos, estrategias, tácticas, metas a corto plazo, tipo de militancia, forma de realizar elecciones internas y un largo etcétera con el fin de mantener la unidad e identidad de la organización. Parte de las estructuras de expectativas de una organización es, de igual modo, la figura de una estructura de cargos o roles - una jerarquía - dentro raizados en la cultura de la organización: ambos plexos - la estructura de posiciones y la cultura organizacional- contienen toda la complejidad de la organización. Sobre la base de la red de expectativas, una organización establece un orden para la toma de decisiones futuras $y$, de esta forma, evita la improvisación: pone en movimiento su cadena de comunicación de decisiones que le confieren unidad e identidad (Luhmann, 2000, p. 417).

\subsection{Estructura de posiciones: jerarquía}

La estructura de roles o posiciones en una organización son decisiones que se han solidificado y han alcanzado una forma específica en ella: un rol o una posición de decisión (Luhmann, 2000, p. 207). Las organizaciones tienen diferentes roles que conforman una red de posiciones o espacios de decisión con formas diferenciadas. En cada organización, se tiene una estructura de posiciones que ha sido erigida desde el momento mismo de la constitución de la organización y que se va adaptando y va cambiando con el tiempo. En cualquier caso, la mayoría de las organizaciones puede cambiar la forma de sus estructuras de posiciones e, incluso, parte de sus estructuras de expectativas. Al mismo tiempo, la estructura de posiciones establece un orden que direcciona la cadena de decisiones hacia un objetivo específico $\mathrm{y}$, a su vez, establece las tareas internas que deben cumplirse para tal fin. La estructura de posiciones le da unidad y estrategia a la organización en términos de sus operaciones. Las decisiones en la organización se 
distribuyen en diferentes secciones de la organización, mientras que la tarea de manejar complejidad y enfrentar riesgos del entorno se distribuye en diferentes secciones. En este sentido, el proceso de toma de decisiones se regula a través del proceso de diferenciación de su estructura en distintas formas: diseños funcionales, departamentales, matriciales. El establecer una jerarquía como la forma principal de coordinación entre los componentes de una organización tiene la función de reducir la complejidad y distribuir el potencial de conflictos y problemas. La estructura de posiciones en la forma de jerarquía especifica la relación de poder desde un centro a la periferia (Luhmann, 2000, p. 211). En organizaciones con una jerarquía altamente estructurada, la mayor parte de las decisiones se realiza, precisamente, en el centro de las organizaciones. Las decisiones que aquí se toman son centrales en la definición del futuro de la organización, pero, al mismo tiempo, producen inflexibilidad y rigidez para enfrentar problemas internos y externos. ${ }^{13}$

\subsection{La cultura organizacional}

Junto a la inflexible estructura de posiciones que le brinda unidad, identidad, coherencia y dirección a la cadena de decisiones que constituyen a las organizaciones sociales, se halla otro umbral en el cual las expectativas $\mathrm{y}$, principalmente, las expectativas de expectativas se consolidan en el sistema y constituyen una red que, ya en la forma de un acervo de conocimiento o ya como un bagaje de normas, crean un mar- co estructural para el proceso de toma de decisiones y para enmarcar las interacciones diarias dentro de las organizaciones. Estos espacios informales dentro de toda organización son necesarios, dada la enorme complejidad interna y porque no todos los mecanismos de control funcionan de manera eficaz en el ambiente interno. Al mismo tiempo, estos espacios informales son necesarios para hacer más compleja la organización y, fundamentalmente, para hacer más complejos a los individuos que son sus miembros. Es, también, un ámbito de aprendizaje y recreación que permite cierta contingencia en las actividades cotidianas, frente a la rigidez de las reglamentaciones internas y la jerarquización de fines y medios. Este acervo de conocimiento, normas y símbolos que constituye la red de expectativas de la organización se llama cultura organizacional (Luhmann, 2000, p. 240). Así, el ambiente interno que emerge como cultura organizacional provee el marco de expectativas para regular las relaciones informales entre sus miembros y constituye, al mismo tiempo, el trasfondo sobre el que se toman las decisiones cotidianas. De suma importancia es decir que, si bien las estructuras informales son necesarias para lograr los objetivos de la organización, fundamentalmente se convierten en un espacio para la innovación y el mejoramiento.

\subsection{La estructura y organización de la protesta}

Hasta aquí, se ha argumentado que las organizaciones sociales que hacen uso re-
13 Una disyuntiva recurrente entre las organizaciones populares de la Ciudad de México fue elegir entre el mecanismo de 'centralismo democrático', que consistía en la toma de decisiones mediante el consenso de un cuerpo selecto de militantes, o el 'mando unico', que consistía en la decisión unipersonal de los 'lideres' o de los 'lideres históricos' de gran parte de estas organizaciones.

\section{territarias 31}

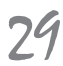


currente de la protesta como estrategia para mantener sus operaciones a través de la absorción de recursos desde su entorno, particularmente, desde el sistema político, deben observarse como cadenas recursivas de decisiones que, a través del tiempo, consolidan una estructura de roles o posiciones y construyen una cultura organizacional propia. Sin embargo, en este punto particular de sus operaciones, es decir, al momento en que las organizaciones hacen uso de la producción y reproducción de comunicaciones orientadas, precisamente, al conflicto, ya sea que esta refiera a valores, identidades u horizontes de sentido alternativos o a temas políticos, económicos o políticos, el problema teórico que en ese momento debe resolverse es ¿̨cómo la comunicación de protesta, una comunicación de suyo conflictiva y problemática, se encadena y estabiliza en el tiempo, conforma estructuras vinculantes y constituye un orden emergente que llega a adquirir la forma de organización en protesta? $\mathrm{O}$, en otras palabras, ¿‘cómo la comunicación de la protesta se convierte en un sistema social autorreferente, operativamente clausurado y autopoiético? En efecto, si la comunicación de la protesta, dado que está orientada al conflicto, no es capaz de producir conexiones vinculantes y no es capaz de conectarse consigo misma en una cadena, desaparece. Por lo tanto, para que la comunicación de la protesta genere un sistema que se autorreproduzca y transforme en el tiempo, es necesario que emerjan una serie de mecanismos que bien vale la pena señalar.
1) En un primer momento, el sistema construye un marco de expectativas sobre la base de la información generada internamente, así como la información que construye acerca de su entorno. Tales variables permiten identificar las condiciones políticas oportunas para iniciar la movilización, la manifestación o el diseño de estrategias a futuro. 2) Además, la identificación oportuna de las posibilidades de movilización muestra un impacto interno sobre la protesta y, hasta cierto punto, influye en la apropiación, configuración y uso de los recursos organizacionales. 3) En efecto, si la protesta encuentra conectividad en amplias redes de comunicación, entonces se amplían los circuitos de provisión de recursos materiales y simbólicos. En todo caso, para impulsar la movilización de la protesta, lo necesario es la articulación estructural de los recursos organizacionales, simbólicos, materiales y humanos, así como la sistematización y anudamiento de los objetivos, estrategias y recursos simbólicos que son fundamentales para mantener el aparente consenso entre los manifestantes. 4) En coyunturas de protesta, las organizaciones militantes ya no solo movilizan recursos materiales, simbólicos y humanos, sino que ahora también movilizan "reasons, commitments and ties” (Luhmann, 1998, p. 850). 5) La movilización, por lo tanto, reconoce, organiza y prepara a los militantes para salir a campo a la manifestación pública del descontento, de los agravios y las demandas. Al hacerlo, las organizaciones seleccionan ciertos espacios urbanos, espacios cargados de simbolismo, que le- 
gitiman los eventos de protesta, los cuales tienen referencias simbólicas y de sentido. 6) La demostración de masas es un mensaje y un medio de comunicación mediante el cual un grupo de la sociedad comunica el rechazo, su protesta y el desafío contra la sociedad y, específicamente, contra su sistema político. Al mismo tiempo, muestra el número, la fortaleza de la organización y la amplitud del descontento. 7) La demostración pública, además, implica otra clase de artefactos comunicativos de la protesta, como la comunicación del miedo, la confianza y el compromiso moral, mediante el uso de medios de difusión, como el lenguaje no verbal, oral, escrito, y medios de masas, como los discursos, las reuniones públicas, las páginas web, los libros, los folletos. 8) Así, se despliegan, también, diversas estrategias y tácticas para expresar su descontento o para la comunicación de la protesta mediante el uso de un repertorio de contención. 9) Igualmente, en medio de sus episodios de protesta, la organización, eventualmente, reconfigura sus propias líneas programáticas e identidades, a través de diversos mecanismos. El programa tal vez evolucione, se desarrolle con nuevo contenido y expanda sus horizontes en un proceso que se conoce en la literatura clásica como un mecanismo de 'enmarcado'; o, incluso, puede suceder que las identidades de la organización se diferencien frente a otras organizaciones y movimiento, y se diferencien de sí mismas. 10) Finalmente, la movilización y la comunicación de la protesta implican la construcción comunicativa de un actor colectivo: de la organización como actor en protesta. Así y solo así, sobre la base de esta ficción colectiva, es posible la comunicación en la esfera de lo público.

\section{Conclusiones}

Diferenciar organizaciones de los movimientos sociales de las organizaciones en protesta es una tarea necesaria para entender las particulares relaciones que estas últimas entablan con su entorno, así como sus dinámicas internas. Un marco teórico general de lo social, como la perspectiva sistémica, aporta las herramientas conceptuales para emprender tal reto y poder observarlas de forma multidimensional. Sin embargo, la propuesta aquí esbozada es, tan solo, un llamado de atención al respecto, más que los lineamientos de un modelo acabado. Falta, por supuesto, profundizar y atender diversas líneas de investigación que se abren desde el horizonte de la teoría de los sistemas sociales, así como profundizar en las necesarias convergencias conceptuales entre las teorías convencionales de los movimientos sociales y su particular tratamiento de las organizaciones, por un lado, y el enfoque sistémico, por el otro. Como traté de demostrar en este manuscrito, la riqueza conceptual resultante de la mutua interrelación entre ambos enfoques solo puede beneficiar a la investigación de lo social en términos de generación de conocimiento empírico.

Por otro lado, la generalidad del enfoque sistémico permite observar múltiples dimensiones de lo social y, en lo particular, de las organizaciones sociales que recurren territarias 31 
a la protesta como forma de operación y comunicación con el sistema político. De lo que se trata, finalmente, es de elaborar esquemas conceptuales que nos permitan elaborar comparaciones trasnacionales que aporten a un acervo de conocimiento sobre las organizaciones. Bien es cierto que las observaciones sobre las organizaciones sociales de la Ciudad de México carecen de un enfoque sistemático que nos permita aventurar cualquier hipótesis de carácter más general, no obstante, tómese este primer esfuerzo como un primer paso en esta dirección. Por último, es importante decir que el giro sistémico permite elaborar estudios no solo de las diversas estructuras de las organizaciones, como lo son las jerarquías y cultura organizacionales, sino que, además, permite estudiarlas en diferentes niveles, como las interacciones dentro de las organizaciones, las relaciones entre organizaciones y entre estas y el sistema político, y, también, permite incorporar dimensiones sociales hasta ahora no determinantes en el análisis. La propuesta que aquí se presentó se encamina en esta dirección.

\section{Referencias}

Abraham, M., \& Büschges, G. (2004). Einführung in die organisationssoziologie. Wiesbaden: vs Verl. für Sozialwiss.

Ahlemeyer, H. W. (1995). Soziale bewegungen als kommunikationssysteme. Opladen: Leske + Budrich.

\section{territarias 31}

México, D.F.: Universidad de la Ciudad de México.

Álvarez Enríquez, L., San Juan Victoria, C., \& Sánchez Mejorada, C. (2006). Democracia y exclusión. Caminos encontrados en la Ciudad de México. México, D.F.: Universidad Nacional Autónoma de México, Centro de Investigaciones Interdisciplinarias en Ciencias y $\mathrm{Hu}-$ manidades.

Calva, J. L. (2001). México, más allá del neoliberalismo. México: Plaza y Janés.

Cansino Ortiz, C. (2000). La transición mexicana, 1977-2000. México: Centro de Estudios de Política Comparada.

Castells, M. (1988). Movimientos sociales urbanos (10 $10^{\mathrm{a}}$ ed.). México, Madrid: Siglo Veintiuno Editores, Siglo Veintiuno de España Editores.

Clegg, S., \& Bailey, J. (Eds.). (2008). International encyclopedia of organization studies 2. Los Ángeles: SAGE Publications Ltd.

Cohen, J. L. (1985). 'Strategy or identity' new theoretical paradigms and contemporary social movements. Social Research, (52), 663-716.

Crespo, J. A. (2003). México, el fin de la hegemonia partidista. México, D.F.: Centro de Investigación y Docencia Económicas.

Davis, G. F., McAdam, D., Scott, W. R., \& Zald, M. N. (2005). Social movements and organization theory. USA: Cambridge University Press.

Estrada Saavedra, M. (2012a). Los muros están hablando: la protesta gráfica de la Asamblea Popular de los Pueblos de

Édgar Guerra BLANCO 
Oaxaca. En M. Estrada Saavedra y R. Millán (Eds.), La teoría de los sistemas de Niklas Lubmanna prueba:horizontes de aplicación en la investigación social en América Latina. México: El Colegio de México y Universidad Nacional Autónoma de México.

Estrada Saavedra, M. (2012b). Protesta social. Tres estudios sobre movimientos sociales en clave de la teoría de sistemas de Niklas Lubmann. México: El Colegio de México.

Estrada Saavedra, M. (2013). Protest systems: outline of a systems model of social movements. In M. Tzaneva (Ed.), Nachtflug der Eule: 150 Stimmen zum Werk von Niklas Lubmann: Gedenkbuch zum 15. Todestag von Niklas Lubmann (8. Dezember 1927 Lüneburg - 6. November 1998 Oerlinghausen). Berlin: LiDi Europe Verlagshaus.

Estrada Saavedra, M., \& Guerra Blanco, E. (2012). Coda. La perspectiva sistémica para el estudio de los movimientos sociales: ‘solo otro giro de tuerca? En Protesta social. Tres estudios sobre movimientos sociales en clave de la teoría de sistemas de Niklas Lubmann (pp. 251270). México: El Colegio de México.

Gabriel, K. (1979). Analysen der organisationsgesellschaft. Ein kritischer vergleich der gesellschaftstheorien Max Webers, Niklas Lubmanns und der phänomenologischen soziologie. Frankfurt/Main (u.a.): Campus.

Guerra Blanco, E. (2012). Protesta a marchas forzadas. El caso del Frente Popular Francisco Villa en la Ciudad de México,
1983-2010. En M. Estrada Saavedra (Ed.), Protesta social. Tres estudios sobre acción colectiva en clave de la teoría de los sistemas sociales de Niklas Lubmann. México: El Colegio de México.

Guerra Blanco, E. (2013a). Protest organisation and protest actors: the case of the Popular Front Francisco Villa (Dissertation submitted to the Faculty of Sociology at Bielefeld University for the degree of Doctor of Philosophy, Dr. phil., Bielefeld).

Guerra Blanco, E. (2013b). Observing protest organizations as social systems: the Popular Front Francisco Villa's media diffusion articulation of political subjectivities among workers. Contention: The Multidisciplinary Journal of Social Protest, 1(1), 75-87.

Guerra Blanco, E. (2014). Utopía y pragmatismo. Enseñanza y aprendizaje en una organización urbana popular. Interface: A Journal for and about Social Movements, 6(1) The pedagogical practices of social movements, 74-98.

Hellmann, K.-U. (1996). Systemtheorie und neue soziale Bewegungen. Identitütsprobleme in der Risikogesellschaft. Opladen: Westdt. Verl.

Hutter, M., \& Teubner, G. (1994). Der Gesellschaft fette Beute. 'Homo juridicus' und 'homo oeconomicus' als kommunikationserhaltende Fiktionen. In P. Fuchs \& A. Göbel (Eds.), Der mensch - das medium der gesellschaft? (pp. 110-145). Ffm.: Suhrkamp.

Japp, K. (1986a). Kollektive Akteure als soziale Systeme? In H.-J. Unverferth territarias 31 
(Ed.), System und selbstproduktion: zur Esrschließung eines neuen paradigmas in den sozialwissenschaften (pp. 166-191). Frankfurt/Basel: Lang.

Japp, K. (1986b). Neue soziale Bewegungen und die Kontinuität der moderne. In J. Berger (Ed.), Die moderne. Kontinuitäten und züsuren (Vol. Sonderbande 4, pp. 311-333). Göttingen: Soziale Welt.

Japp, K. (1993). Die form des protests in den neuen sozialen bewegungen. In D. Baecker (Ed.), Probleme der form (pp. 230-251). Frankfurt: Suhrkamp.

Jenkins, J. C. (1983). Resource mobilization theory and the study of social movements. Social Research, (9), 527-553.

Kornhauser, W. (2008). The politics of mass society. New Brunswick, New Jersey: Transaction Publishers.

Laraña, E. (2007). La construcción de los movimientos sociales. Madrid: Alianza Editorial.

Luhmann, N. (1964). Funktionen und folgen formaler organisation. Berlin: Duncker \& Humblot.

Luhmann, N. (1975). Interaktion, organisation, gesellschaft. In N. Luhmann, Soziologische aufklärung 2. Aufsätze zur theorie der gesellschaft (pp. 9-20). Opladen: Westdeutscher Verlag.

Luhmann, N. (1977). Differentiation of society. Canadian Journal of Sociology, (2), 29-53.

Luhmann, N. (1981). Organisation im wirtschaftssystem. In N. Luhmann, Soziologische aufklärung 3. Soziales system,

\section{territarias 31}

34
Luhmann, N. (1987). Soziale systeme: Grundrißeiner allgemeinen theorie. Frankfurt am Main: Suhrkamp Verlag.

Luhmann, N. (1996). Protest: systemtheorie und soziale Bewegungen ( $3^{\text {rd }}$ ed.). Frankfurt am Main: Suhrkamp Verlag.

Luhmann, N. (1998). Die gesellschaft der gesellschaft. Frankfurt am Main: Suhrkamp Verlag.

Luhmann, N. (2000). Organisation und entscheidung. Opladen/Wiesbaden: Westdeutscher Verlag.

Luhmann, N. (2003). Soziologie des risikos. Rieden/Allgäu: Gruyter.

Luhmann, N. (2004). Ökologische kommunikation: Kann die moderne gesellschaft sich auf ökologische gefährdungen einstellen? Wiesbaden: vs Verlag für Sozialwisssenschaften.

Luhmann, N. (2005). Organisation und entscheidung. In Soziologische aufklärung 3. Soziales system, gesellschaft, organisation. Wiesbaden: vs Verlag für Sozialwissenschaften.

Luhmann, N. (2007). La sociedad de la sociedad. México: Universidad Iberoamericana/Herder.

March, J. G., \& Simon, H. A. (1958). Organizations. New York: Wiley.

Mayntz, R. (1971). Soziologie der organisation. Hamburg: Rowohlt.

McAdam, D., Tarrow, S., \& Tilly, C. (2001). Dynamics of contention. New York: Cambridge University Press.

McCarthy, J. D, \& Zald, M. N. (1990). Resource mobilization and social movements: a partial theory. In M. N. Zald \& J. D. McCarthy (Eds.), Social move- 
ments in an organizational society: collected essays (pp. 15-48). New Brunswick: Transaction Publishers.

Morris, A. (2000). Reflections on social movement theory: criticisms and proposals. Contemporary Sociology, (29), 445-454.

Nassehi, A. (2005). Organizations as decision machines: Niklas Luhmann's theory of organized social systems. The Sociological Review, (53), 178-191. Retrieved October 1, 2012.

Olvera Rivera, A. (1998). Cambios en los patrones de acción colectiva y el nuevo asociativismo en México. México: Instituto de Investigaciones Histórico-Sociales, Universidad Veracruzana.

Olvera Rivera, A. (2001). Movimientos sociales prodemocráticos, democratización y esfera pública en México. El caso de Alianza Cívica. Xalapa: Universidad Veracruzana, Instituto de Investigaciones Histórico-Sociales.

Olvera Rivera, A. (2004). Civil society in Mexico at century's end. In K. J. Middlebrook (Ed.), Dilemmas of political change in Mexico (pp.403-439). London, San Diego: University of London-Institute for the Study of the Americas, University of California, Center for U.S. Mexican Studies.
Paladino, M. (2010). Intermedicación clientelar de demandas sociales y movilización politica. La vivienda social en la Ciudad de México (Tesis Doctoral, Facultad Latinoamericana de Ciencias Sociales, Sede Académica México).

Perrow, C. (1991). A society of organizations. Theory and Society, (20), 725762.

Porta, D. D., \& Diani, M. (2005). Social movements: an introduction (Revised). Padstow, Cornwall: Blackwell Publishing.

Presthus, R. V. (1962). The organizational society. New York: Vintage Books.

Ramírez Sáiz, J. M. (1987). Política urbana y lucha popular. México, D.F.: Universidad Autónoma Metropolitana.

Simon, H. A. (1991). Organizations and markets. Journal of Economic Perspectives, (5), 25-44.

Smelser, N. J. (1966). Theory of collective behavior. New York: Free Press.

Turner, R. H., \& Killian, L. M. (1972). Collective behaviour. Englewood Cliffs, N.J.: Prentice Hall.

Whyte, W. H. (2002). The organization man ( $1^{\text {st }}$ ed.). Philadelphia: University of Pennsylvania Press. territarios 31

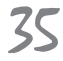

